\title{
Editoriali
}

\section{Epidemiologia del farmaco in psichiatria}

\author{
GIANNI TOGNONI
}

\section{PREMESSA}

Forse il contesto di riferimento più diretto per questo editoriale è un fatto culturale (ed editoriale) che è avvenuto fuori della psichiatria: una rivista tra le più antiche dedicata agli aspetti epidemiologici della medicina, il Journal of Clinical Epidemiology (che aveva segnalato anni prima l'evoluzione metodologica di questo settore, abbandonando il titolo di Journal of Chronic Disease) ha introdotto formalmente nel 1991 una sezione dedicata alla farmacoepidemiologia.

$\mathrm{Si}$ concludeva in questo modo un assestamento, nominale e culturale, che aveva visto il progressivo trasformarsi dell'attenzione occasionale ai patterns di uso dei farmaci nell'una o nell'altra branca della medicina (Bergman et al., 1979) in una vera e propria disciplina (Lawson, 1984), che in pochi anni ha visto addirittura il sorgere di riviste ad hoc (van Boxtel, 1991; Mann, 1992).

Al cuore di questa evoluzione (al di là della nota tendenza "patologica» della letteratura a creare termini diversi ed organi separati per favorire le pubblicazioni rapide di articoli che celano spesso la loro irrilevanza nel trovare ospitalità in nuove riviste «specialistiche») stanno una constatazione di fatto ed una ipotesi metodologica (Tognoni \& Laporte, 1984):

a) i farmaci sono talmente ubiquitari e protagonisti del fare medico, da esigere una considerazione specifica con studi descrittivi sistematici;

b) in quanto espressione concreta (per quanto spesso implicita) del processo conoscitivo e decisionale, diagnostico e prognostico, dell'agire medico, i farmaci (non tanto il loro numero e la loro frequenza, ma la logica della loro prescrizione o meno) sono indicatori privilegiati dell'interazione tra i fattori più

\footnotetext{
Indirizzo per la corrispondenza: Dr. G. Tognoni, Laboratorio di Farmacologia Clinica, Istituto di Ricerche Farmacologiche Mario Negri, Via Eritrea 62, 20157 Milano.

Fax $(+39)$ 02-354.6277
}

strettamente clinici e quelli sociali e culturali che determinano il profilo di beneficio-rischio e di beneficiocosto degli interventi medici nel loro complesso.

La differenza delle due prospettive non è irrilevante:

a) il farmaco «descrittore» ha una sua vita autonoma, è l'oggetto di studi di drug utilization, che documentano l'andamento della prescrizione nei contesti di cura più diversi (ospedalieri e ambulatoriali), la sua intensità, variabilità, appropriatezza o meno rispetto a generici standard attesi, di comportamento o di mercato;

b) il farmaco «indicatore» è trattato come una vera e propria variabile epidemiologica, non leggibile (o ben scarsamente informativa, o addirittura confondente) se non nella sua relazione con la storia dei problemi e delle popolazioni.

Lo studio del suo uso avrà interesse in proporzione:

- al ruolo più o meno rilevante che gioca nel determinare l'andamento e l'esito delle patologie per le quali è stato prescritto (o che può avere causato);

- alla sua funzione di «rivelatore» di nodi e causalità culturali $e / o$ istituzionali che stanno dietro il dato prescrittivo (Tognoni et al., 1991).

Gli studi che descrivono sono essenziali per i settori di mercato ad alto turnover, in cui l'informazione è tutta nelle modificazioni e nelle variabilità quantitative (Montanaro et al., 1992) e/o nelle implicazioni importanti di spesa (Haayer-Ruskamp \& Dukes, 1986): diventano irrilevanti dove il quesito riguarda il se e quanto la razionalità o meno di strategie terapeutiche comporta modificazioni epidemiologiche della morbi-mortalità.

La prospettiva $b$ ) coincide con l'epidemiologia analitica e clinica del farmaco e abbraccia dalla sperimentazione clinica, alla post-marketing surveillance, agli studi valutativi di esito, alle meta-analisi: tutto ciò che sta tra l'epidemiologia dei problemi-malattia e quella dell'impatto epidemiologico dei trattamenti (Tognoni \& Laporte, 1992).

La storia di queste due prospettive è ormai larga- 
mente documentata nella letteratura ed ha portato alla polarizzazione sempre più evidente tra atteggiamenti descrittivo-amministrativi (valga per tutti il recentissimo numero della rivista ufficiale di farmacologia e terapia USA) (Workshop on drug utilization, 1991) ed epidemiologici-valutativi (Terzian \& Tognoni, 1991; Lunde, 1992; Romero et al., 1991).

La "premessa» può essere sembrata troppo lunga: di fatto porta in sé anche tutto quanto c'è da dire specificamente sulla epidemiologia del farmaco in psichiatria.

\section{IL CONTESTO}

La psichiatria è l'area modello del non-divenire per quello che riguarda i trattamenti, almeno nel loro rapporto con l'andamento e l'esito dei problemi e delle popolazioni.

È nello stesso tempo l'area degli incessanti movimenti browniani delle novità apparenti o proclamate, che occupano nicchie diagnostico-terapeutiche più o meno incerte ed epidemiologicamente indefinite, nell'attesa mai stanca di futuri risolutivi punti di attacco biochimico-funzionale per nuove generazioni di farmaci (Unità di Psichiatria, 1986-1992).

L'attenzione più intelligente è chiaramente sul contesto di assistenza.

I trattamenti farmacologici sono inevitabilmente destinati ad essere una variabile che sembra cambiare, ma solo per aggiornarsi cosmeticamente.

\section{LE IMPLICAZIONI}

«Descrivere» l'uso dei farmaci in psichiatria significa destinarsi ad essere ripetitivi ed epidemiologicamente irrilevanti: ad «osservare» ciò che è perfettamente «atteso», cioè che ci sono variabilità, quantitativamente più o meno impressionanti, ma che non informano sul perché e sul come della variabilità, e che soprattutto rimandano al sospetto di fondo che tale variabilità sta con l'esito dei problemi (se c'erano) e dei pazienti (sostituiti da medie e stime) in un rapporto casuale.

Gli studi di utilizzazione dei farmaci (con le loro stime, percentuali, numero di farmaci per paziente, ranges, $\mathrm{p} \leq$, abbelliti di elaborazioni statistiche più o meno «eleganti») forse sono, scientificamente, del genere "C'era una volta...».

Utili, ogni tanto, come esercizio pre-post laurea, e come tali, di tanto in tanto pubblicabili, anche perché sempre capaci di evocare discussioni che giustap- pongono interpretazioni per le quali non sono stati raccolti dati, e di produrre cross-tabulazioni, che mimano popolazioni (schizofrenici, primi casi, cronici, donne depresse menopausali, utenti urbani, rurali, alta bassa scolarizzazione, ecc.) (de Girolamo et al., 1987).

È ogni tanto utile, e necessario, per un Paese, o per una USL, o per una istituzione, fotografarsi rispetto all'uso dei suoi farmaci psichiatrici: ma sapendo che è una foto-tessera, non è il percorso di ricerca (hard e soft) proposto da un film come Blow-up.

\section{COME MAI?}

La psichiatria non ha sfruttato il suo essere candidata naturale a non usare il farmaco come descrittore, ma a vederne la funzione di «indicatore».

La possibilità e le implicazioni di una scelta tra i due approcci erano già state perfettamente formulate più di 10 anni fa (Tognoni et al., 1981) in un confronto che includeva la descrizione, la valutazione clinica controllata, l'epidemiologia clinica e quella istituzionale.

$\mathrm{Da}$ allora le cose «intelligenti» sul ruolo del farmaco in psichiatria (e la sua epidemiologia) non sono più passate per studi di uso di farmaci, o per le tabelle che ogni tanto aggiornano ragionieristicamente la lista delle nuove pubblicazioni, ma per ricerche che approfondivano e verificano gli altri protagonisti di quel confronto, con un ampliamento importante alla medicina di base (Holm \& Olesen, 1988).

\section{CHE FARE?}

Sarebbe di cattivo gusto riprendere cose già dette altrove, e ben corredate di bibliografia, non troppo vecchia (Saraceno, 1990), anche perché nulla di sostanzialmente nuovo è successo (Unità di Psichiatria, 1986-1992).

Si tratta in fondo per la psichiatria di prendere sul serio, nella definizione che qualifica l'area di interesse di questo editoriale, il termine epidemiologia più di quello di farmaco, possibilmente avendo in mente la variante clinica della epidemiologia stessa, quella che non solo descrive le associazioni più o meno causali, ma ne indaga i perché, ne verifica la evitabilità, coinvolge $i$ responsabili, dialoga intensivamente con gli operatori.

Le referenze citate nella premessa possono essere metodologicamente pertinenti (Tognoni et al., 1991; Terzian \& Tognoni, 1991; Lunde, 1992). 


\section{PER NON AVER TIMORE DEI PERCORSI ATIPICI}

C'è un libro recente, che racconta la storia di un classico dell'epidemiologia degli psicofarmaci (della psichiatria?), la relazione tra benzodiazepine e dipendenza (Medawar, 1992).

Potrebbe non essere male usarlo (anche se è un racconto) come un buon testo di metodologia (con il vantaggio di non essere noioso), per uno sviluppo creativo della epidemiologia del farmaco.

Parla di storia e di poteri, di mercato e di istituzioni, di innovazioni scientifiche e di promesse terapeutiche, di operatori e pazienti, delle loro interazioni, contrapposizioni, rapporti: un vero e proprio «scenario" epidemiologico.

Proprio quello che serve anche in psichiatria (fatte le debite equivalenze tra attori e variabili) per non permettere più al farmaco di essere un descrittore.

\section{BIBLIOGRAFIA}

Bergman U., Grimsson A., Wahba A. H. W. \& Westerholm B. (ed.) (1979). Studies in Drug Utilization. Methods and Applications. WHO Regional Office for Europe: Copenhagen.

van Boxtel C. J. (ed.) (1991). Post Marketing Surveillance. Elsevier Science: Amsterdam.

de Girolamo G., Williams P. \& Cappiello V. (1987). Psychotropic drug utilization and audit in two Italian Psychiatric services. Psychological Medicina, 17, 989-997 (Vedi specificamente la Tabella comparativa degli studi pubblicati).

Haayer-Ruskamp F. M. \& Dukes M. N. G. (1986). Drugs and Money. A preliminary Survey and Research Proposal. WHO: Groningen (Disponibile come n. 53-54 nei Quaderni di Sanità Pubblica, CSI: Milano).
Holm M. \& Olesen F. (1988). Factors affecting prescription of psychotropic drugs in general practice. Scandinavian Journal of Primary Health Care 6, 169-173.

Lawson D. H. (1984). Pharmaco-epidemiology: a new discipline. British Medical Journal 289, 940-941.

Lunde P. K. M. (1992). L'uso razionale dei farmaci. Ricerca \& Pratica 43, 24-36 (Traduzione di un testo ancora in press presentato nel Seminario Internazionale «Auditing Drug TherapyApproaches toward rationality at reasonable cost',, Stoccol$m a, 1991)$.

Mann R. (ed.) (1992). Pharmacoepidemiology and Drug Safety. John Wiley: West Sussex.

Medawar C. (ed.) (1992). Power and Dependence. Social Audit on the safety of medicines: London (Commentato per le implicazioni metodologiche sul significato di epidemiologia in Ricerca \& Pratica 45, 92-98).

Montanaro M., Magrini N., Vaccheri A. \& Battilana M. (1992). Drug utilization in general practice: prescribing habits of National Formulary drugs by GPs of Emilia Romanga (Italy) in 1988 and 1989. European Journal of Clinical Pharmacology $42,401-408$.

Romero M., Giambuzzi M., Nigro et al. (1991). DURG-I: una bibliografia e qualcosa di più. Giornale Italiano di Farmacia Clinica 5, 41-49.

Saraceno B. (ed.) (1990). Uso Razionale degli Psicofarmaci. Farmacie Comunali Riunite: Reggio Emilia.

Terzian E. \& Tognoni G. (1991). An epidemiological framework for essential drugs. ISDB Review 1, 19-24.

Tognoni G. \& Laporte J.R. (ed.) (1984). Epidemiologia del Farmaco. Il Pensiero Scientifico: Roma (Vedi soprattutto il primo capitolo pp. 1-33 e l'ultimo capitolo pp. 157-169).

Tognoni G. \& Laporte J.R. (1992). From clinical trials to drug utilization studies. In Drug Utilization Studies: Methods and Use. WHO: Copenhagen (in press).

Tognoni G., Bellantuono C. \& Lader M. (ed.) (1981). Epidemiological Impact of Psychotropic Drugs. Elsevier: Amsterdam.

Tognoni G., Martini N. \& Del Favero A. (1991). Appunti per ricordare giugno 1991. Giornale Italiano di Farmacia Clinica $5,81-86$.

Unità di Psichiatria, Istituto di Ricerche Farmacologiche «Mario Negri» (1986-1992). Lettera. Percorsi Bibliografici in Psichiatria, volumi I-XIII, 1986-1992 (Vedi soprattutto: volume IV, 1-27, 1987; volume XI, 1991, tutto).

Workshop on drug utilization review. Rockville, Maryland, November 26-27, 1990. Clinical Pharmacology \& Therapeutics 50, Part 2, 1991. 
